\title{
Monitoring morphology and properties of hybrid organic- inorganic materials from in situ polymerization of tetraethoxysilane in polyimide polymer: 1. Effect of the coupling agent on the microstructure and interfacial interaction
}

\author{
Hamid Kaddami, ${ }^{1 *}$ Carsten Becker-Willinger, ${ }^{2}$ Helmut Schmid, ${ }^{2}$
}

1 University CADI AYYAD, Faculty of Science and Technics-Guéliz, Avenue Abdelkrim Elkhattabi, B.P. 549, Marrakech, Morocco. Fax (212)-44- 433170, e-mail: hkaddami@fstg-marrakech.ac.ma

2 Leibniz-Institut für Neue Materialien gem GmbH, Im Stadtwald, Geb. 43, D-66123 Saarbrücken, Germany. Fax (49) -681- 9300271, e-mail: Carsten.BeckerWillinger@inm-gmbh.de

(Received: 19 October, 2005; Published: 2 May, 2006 )

\begin{abstract}
Transmission electron microscopy (TEM), small angle X-ray (SAXS) and dynamical mechanical thermal analysis (DMTA) were used to characterize the morphology and thermo-mechanical properties of hybrid organic inorganic materials. These materials were based on polyimide $(\mathrm{PI})$ and tetraethoxysilane (TEOS). Polyimide polymer is prepared from 4,4'-oxydianiline (ODA) 2,2-Bis (3amino-4-hydroxyphenyl) hexafluoro-propane (6F-OHDA) pyromellitic dianhydride (PMDA) polyamic polymer. In one family of hybrid materials 3isocyanatopropyltriethoxysilane (ICTS) is used as coupling agent in order to enhance the interfacial interaction between polyimide and silica. It was possible to modulate the morphology as well as the optical and thermo-mechanical properties of these hybrid materials depending on the formulation used. TEM and SAXS analysis indicated that silica domains on the nanoscale level are obtained when coupling agent is used in the formulation. Additionally the TEM and SAXS analysis indicated that miscibility of the organic and the inorganic phases on the molecular scale is obtained in the hybrid films when ICTS as coupling agent is added to the polyamic acid. These techniques show a fractal structure of the hybrid materials with coupling agent. This was confirmed with DMTA analysis which shows very high temperature relaxation (more than $450^{\circ} \mathrm{C}$ ). From this result it could be derived that the addition of ICTS causes a morphological transformation from discrete particulate microstructure to fine interpenetrated or co-continuous phases. The intimate miscibility of the phases is accompanied at the same time by the amelioration of thermo-mechanical properties of the hybrid films.
\end{abstract}

\section{Introduction}

Because of their excellent thermal stability, electric insulation ability, physical and mechanical properties, polyimide materials have been extensively used in the aerospace and electronic fields [1] in the past. The applications of polyimides include insulating or dielectric layers, passivation layers, etc [2]. They require good mechanical and physical properties, especially a low specific thermal expansion. In electric applications an enormous heat may be generated and the mismatch of the 
thermal expansion coefficient between metal and electric insulation layer could induce a catastrophic destruction of the multilayer accompanied by a complete loss of the insulation properties and failure of complete components.

The incorporation of inorganic material into the polyimide is one promising method to overcome the above mentioned problems. Silica $\left(\mathrm{SiO}_{2}\right)$ which exhibits very low values of water uptake (close to $0 \mathrm{wt} \%$ ) and low coefficient of thermal expansion (ca $5.10^{-7} \mathrm{~K}^{-1}$ ), would be more suited for microelectronic applications though its dielectric properties and polarisation are inferior to polyimide. Another drawback of silica is the high temperature required for the densification of appropriate layers and also the low flexibility compared to polyimides which certainly limits the use of such kind of materials. The formation of organic-inorganic hybrid materials however may exhibit a combination of favourable properties of both polyimide and silica. The classical combination of both organic and inorganic components (filling, composite) however leads to heterogeneous products thus limiting the use of such type of materials.

In recent years, numerous studies have been reported on hybrid organic-inorganic materials which use the well known polymerization (hydrolytic condensation) of silicon or titanium alkoxides [3-12]. This synthesis via the so called sol-gel approach enables to incorporate inorganic networks into organic polymeric materials at moderate temperatures. It also provides connectivity between organic and inorganic phases, either by in-situ precipitation of $\mathrm{SiO}_{2}$ particles into elastomers or by hydrolysis and condensation of inorganic alkoxides, generally tetraethoxysilane (TEOS), within organic thermoplastic polymers. The final microstructure of the organic-inorganic materials does not only depend on the kinetics of the hydrolysis and condensation of the alkoxides, but also on the strength of interactions between the two phases. The sol-gel method of preparing hybrid materials offers the possibility to control the interfacial interactions by carefully choosing an appropriate coupling agent. Good interfacial interactions lead to considerable mechanical and thermal properties of the resulting materials.

In this work high molecular weight polyimide/silica based hybrid materials, with and without extensive chemical bonding between the two phases has been explored. The chemical bonding between organic and inorganic phases has been performed using isocyanatopropyltriethoxysilane (ICTS) as coupling agent. The purpose was to compare the morphologies of the created compositions studied by small angle X-ray scattering (SAXS) and transmission electron microscopy (TEM) between the hybrid systems with and without ICTS and to combine them with the thermo-mechanical properties investigated by DMTA studies.

\section{Result and discussion}

According to previous description the polyamide matrices have been synthesised from pyrromellitic dianhydride (PMDA) and a mixture of aromatic diamines 4,4'Oxydianiline (ODA) and 2,2-Bis(3-amino-4-hydroxyphenyl) hexafluoro-propane (6FOHDA) in a molar ratio ODA / 6F-OHDA 90:10. The monomer 6F-OHDA has been chosen, because it contains two additional hydroxyl groups which enable to act as optional cross linking point in case of presence of an appropriate co-reactant such as e.g. silane coupling agent. The silica structures used for the formation of hybrid structures have been derived from in situ hydrolysis and condensation of tetraethoxysilane (TEOS) in presence of the polyamic acid. Two routes have been followed in order to provide different connectivity's in the resulting networks and to 
establish different compatibilisation between the organic and the inorganic phases resulting in different morphologies with respect to the microstructure. The first route only comprises of the organic matrix as described above and silica from TEOS. In the second route isocyanatopropyltriethoxysilane (ICPTES) as silane coupling agent has also been added in order to provide adhesion between the organic and the inorganic phase. Table 1 shows the composition of the studied hybrid films along with their densities. The evolution of the density in dependence on the calculated silica content indicates that the hydrolytic condensation and the polyamide formation should be almost complete. Additionally it should be noted that except the PI-ICTS-Silica film which is slightly opaque, all PI-ICTS-Silica films are transparent indicating that macroscopic phase separation has not occurred, which suggests that nanocomposites could be obtained up to $30 \mathrm{wt} \%$ of silica. On the other hand all PISilica hybrid films without the isocyanatosilane coupling agent are opaque indicating the occurrence of micro scale phase separation. The positive effect of the coupling agent ICTS in that direction is then clearly observed. In fact, it reduces the interfacial free energy and at the same time reduces the size of silica particles by strengthening the interactions between TEOS and Polyimide matrix. On the other hand the densities of the PI-ICTS-Silica hybrid films are slightly lower than those of the PISilica hybrid films with the same silica content. This indicates a slightly more compact structure of PI- Silica hybrid films, which mainly caused by the micro scale phase separated morphology. On the other hand, the fragility of PI-ICTS-Silica hybrid materials is significantly increased in particular when higher contents of silica are introduced (more than 15 weight percent). Similar behaviour and properties have been obtained for hybrid organic inorganic materials based on PMMA [9], PVAc [10], Polyurethane [4], etc.

Tab 1. Composition and preparation recipes of the studied hybrids.

\begin{tabular}{|c|c|c|c|c|c|c|}
\hline $\begin{array}{c}\text { Sample } \\
\text { Identification }\end{array}$ & $\begin{array}{l}\text { PAA } \\
\text { (g) }\end{array}$ & $\begin{array}{l}\text { TEOS } \\
\text { (g) }\end{array}$ & $\begin{array}{l}\text { Silica } \\
\% \text { (a) }\end{array}$ & $\frac{n(\text { ICTS })}{n(6 F-\text { OHDA })}$ & $\begin{array}{l}\text { Remark } \\
\text { (b) }\end{array}$ & $\begin{array}{l}\text { Density } \\
\left(\mathrm{g} / \mathrm{cm}^{3}\right)\end{array}$ \\
\hline PI-Silica-0 & 6 & 0 & 0 & 0 & $\mathrm{~T}$ & 1.415 \\
\hline Pl-Silica-05 & 6 & 1.09 & 5 & 0 & O-T & 1.435 \\
\hline PI-Silica-10 & 6 & 2.31 & 10 & 0 & 0 & 1.450 \\
\hline PI-Silica-15 & 6 & 3.67 & 15 & 0 & $\mathrm{O}$ & 1.476 \\
\hline PI-Silica-30 & 6 & 8.92 & 30 & 0 & 0 & 1.521 \\
\hline PI-ICTS-Silica-0 & $\overline{6}$ & 0 & 0 & 1.5 & $\bar{T}$ & 1.411 \\
\hline PI-ICTS-Silica-05 & 6 & 1.09 & 5 & 1.5 & $\mathrm{~T}$ & 1.430 \\
\hline PI-ICTS-Silica-10 & 6 & 2.31 & 10 & 1.5 & $\mathrm{~T}$ & 1.446 \\
\hline PI-ICTS-Silica-15 & 6 & 3.67 & 15 & 1.5 & $\mathrm{~T}$ & 1.470 \\
\hline PI-ICTS-Silica-30 & 6 & 8.92 & 30 & 1.5 & O-T & 1.518 \\
\hline
\end{tabular}

(a) the silica contents are calculated by assuming that sol-gel reaction was almost completed.

(b) T- transparent, O- opaque. 


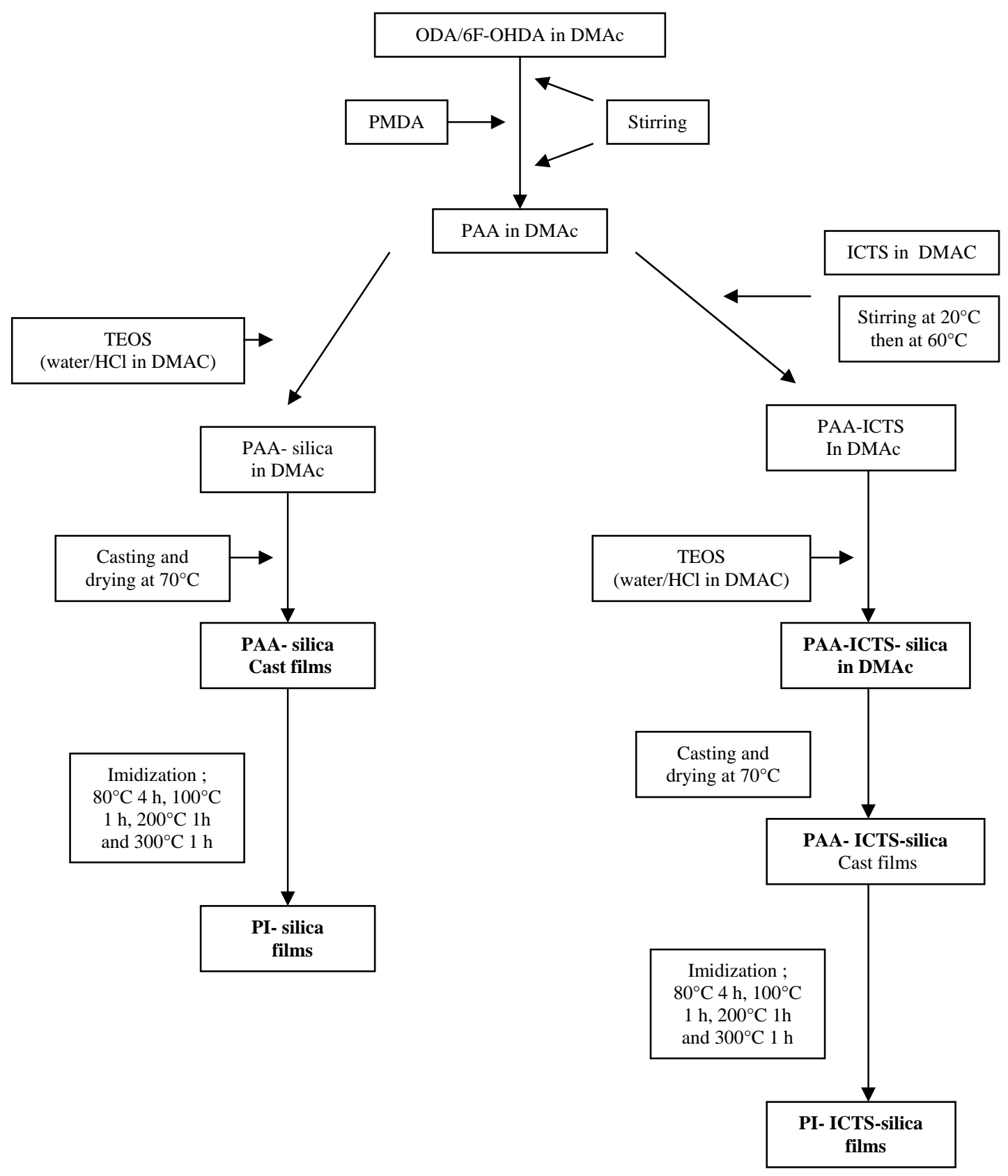

Fig. 1. Schematic diagram method of PI-silica and PI-ICTS-silica films

These observations could also be confirmed by TEM analysis, presented in Figures 2 and 3 respectively. For the systems PI-Silica-15 and PI-ICST-Silica-15 as representative examples for all other hybrid systems, direct comparison of the TEM micrographs indicates that the microstructures are completely different. For the system PI-Silica-10 large particles up to $1.5 \mu \mathrm{m}$ in diameter are visible. However it's difficult to distinguish any silica particles for the PI-ICTS-Silica hybrid film even on the nanometre scale. This difference relates as mentioned above to the compatibilising effect of the isocyanatosilane. On the other hand the TEM micrograph of the PISilica-10 illustrates that the adhesion of the particles to the polyimide matrix seems to be rather poor as here detachment of the particles from the matrix occurring during 
the TEM sample preparation process is visible. This is due to the poor interactions between the silica and the polymer phase and to the hydrophobic character of the polyimide matrix itself. On the other hand, from the TEM micrograph of the PI-ICTSSilica-10 hybrid film one can see the miscibility between the two phases for the hybrid films by the addition of ICTS to the polyamic acid. The size of inorganic domain is very small, according to TEM lower then $2 \mathrm{~nm}$ and can not be clearly distinguished by this method. It seems that the silica has been "covered" by the polymeric phase. Since ICTS is bound to the polymer backbone, silica can grow along the polymer backbone before the imidization of the polymeric acid. So the addition of ICTS causes a morphological transformation from a microstructure with dispersed particles to fine interpenetrated or co-continuous phases. The polysilicate phase grown along the polymer chain and silica is no longer able to aggregate and form big size silica domains because the ICTS decreases the interfacial free energy part in the whole energy of the system thus allowing the system to contain more interfacial area between the organic and the inorganic phases. This confirms the hypothesis of the formation mechanism of interpenetrated phases.

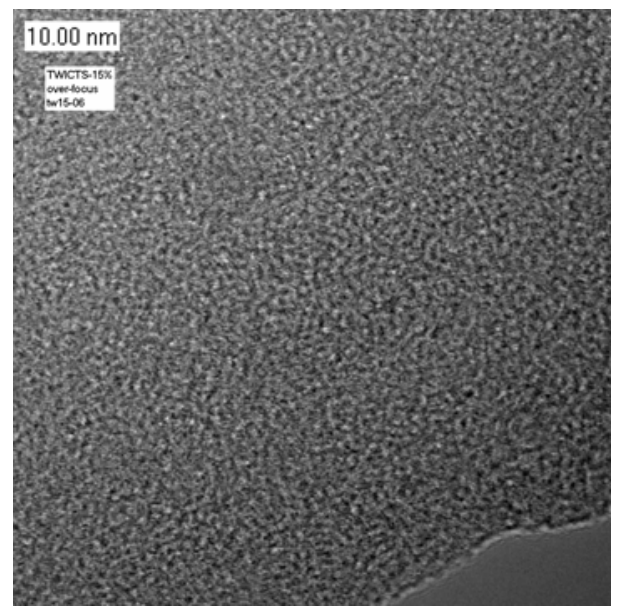

Fig. 2. TEM micrograph of a PI-ICTS-Silica-15.

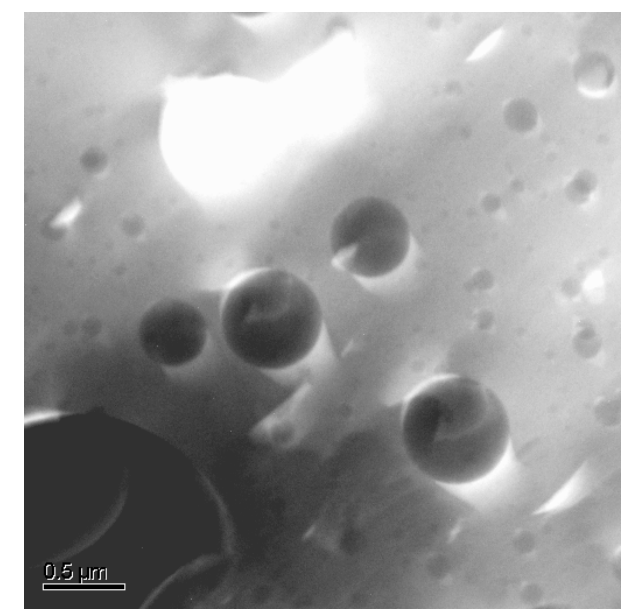

Fig. 3. TEM micrograph of a PI-Silica-15. 
These differences in morphology between the hybrid materials with and without coupling agent ICTS should also be manifested in the SAXS data. Figures 4 and 5 show the logarithmic scattering intensity $\operatorname{Ln}(\mathrm{I})$ on the logarithmic scattering vector In (q) as determined from the SAXS experiments on samples with and without coupling agent ICTS, respectively.

Except the film PI-Silica-5, practically no scattering is shown for PI-Silica hybrid films (without coupling agent) and may be explained by the large size silica domains as observed in TEM micrographs (Figure 4). This observation is in agreement with the TEM results for these films, which show large silica particles in the size range above $100 \mathrm{~nm}$. On the other hand, one can observe a large correlation (broad peak) in SAXS spectra of the hybrid films with coupling agent ICTS which is absent in the hybrid systems without coupling agent ICTS. The existence of such peak confirms the morphology already described above and justifies the existence of a specific nanostructure. In fact the peak indicates the phase separation on the nanometre scale already discussed above.

The $\operatorname{Ln}(\mathrm{I})$-Ln(q) plots show for all films PI-ICTS-Silica a linear region at higher values of the scattering vector $q$. The slopes of 1.2 obtained indicate a fractal structure of the scattering domains. This again confirms the interpenetrated phase structure mentioned above. In such morphology the silica phase could be viewed as a significantly branched structure intimately mixed with the organic polymer (existence of mixed phase). This phenomenon has also been reported by Hajji et al [11] on PHEMA/TEOS based hybrid organic/inorganic materials.

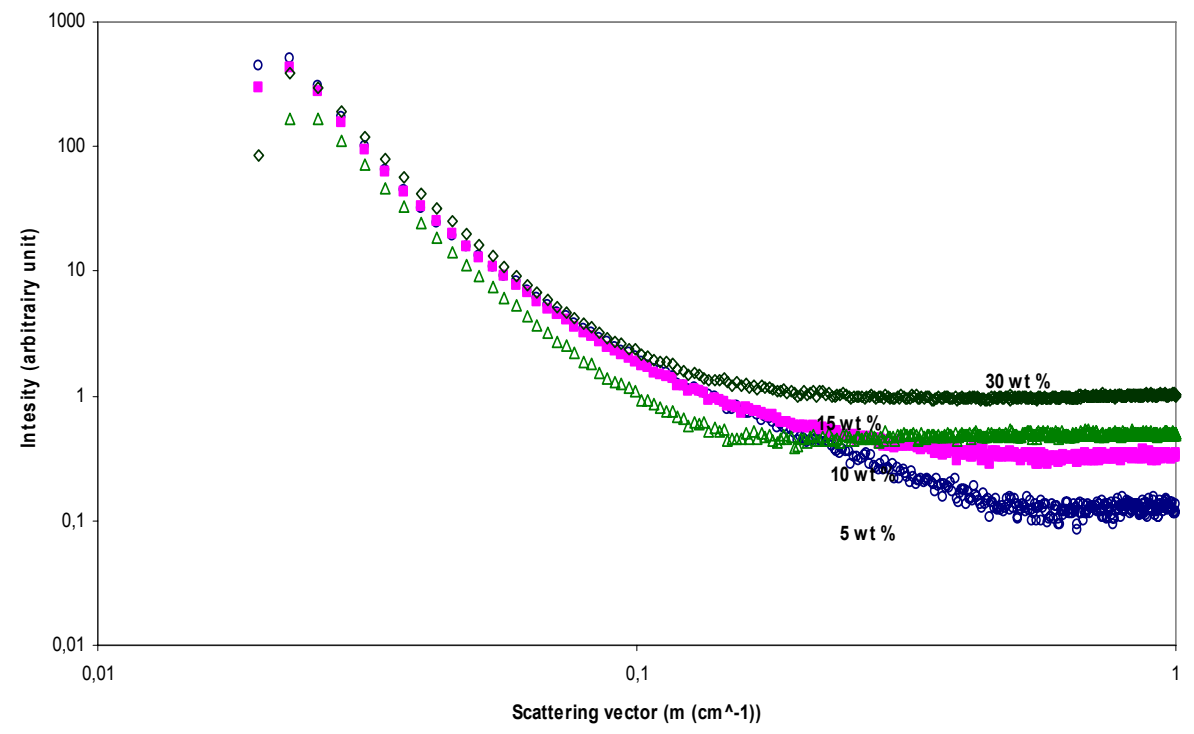

Fig. 4. SAXS spectra of PI-Silica hybrid films for different silica weight fractions. 


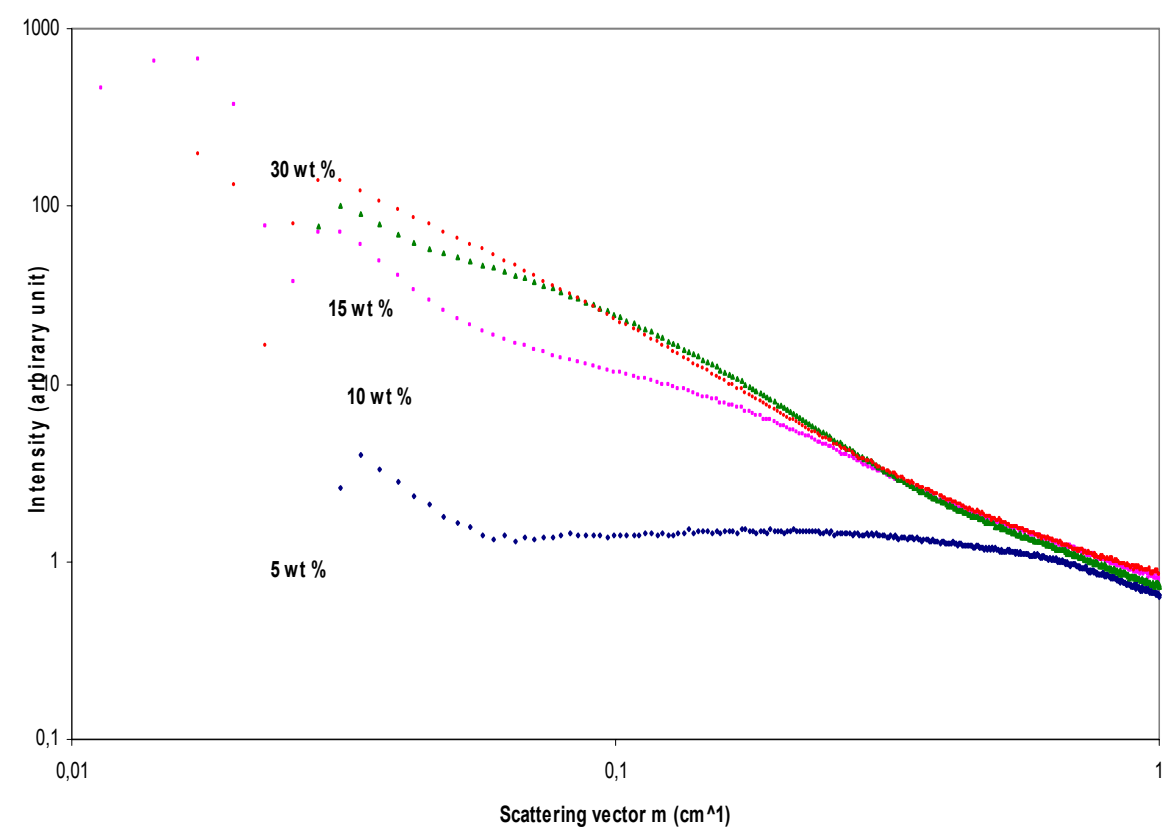

Fig. 5. SAXS spectra of PI-ICTS-Silica hybrid films for different silica weight fractions.

Viscoelastic properties of the pure matrix of the two hybrid families: PI-Silica-00 and PI-ICTS-Silica-00 are presented in Figure 6 and Table II. Only one relaxation, noted $\alpha 1$, is observed in the tan $\delta$ curve for the matrix without coupling agent ICTS and can be attributed to the motions of polyimide main chains. On the other hand two relaxations can be observed for the matrix with coupling agent ICTS. The first relaxation at lower temperature, noted $\alpha 1$, can again be attributed to the motions of polyimide chains, and the relaxation at higher temperature $\left(T \approx 480^{\circ} \mathrm{C}\right)$, noted $\alpha 2$, can be explained by the presence of highly crosslinked and intimately mixed domains, containing both organosilicate (coupling agent) and polyimide chains. Such mixed phases have been reported for many hybrid organic inorganic materials in the literature. Their formation does not only depend on the compatibility (interactions) between organic and inorganic phases but also on the reaction kinetics and the differences in reaction kinetics between organic and/or inorganic polymerization [6, 13].

The lower temperature value of a1 relaxation obtained for the hybrid film with coupling agent ICTS could be explained by the plastification phenomenon created by the ICTS bonded to the polymer backbone. The silane could form side groups along the polymer main chain by direct reaction between the isocyanate group of the ICTS and the carboxylic acid groups of the polyamic acid thus increasing the mobility of the polymer main chain by avoiding chain alignment to occur. On the other hand the slightly higher value of the rubbery modulus of the PI-ICTS-Silica-00 above Tg could be explained by the crosslinking effect of such ICTS groups indicating the transformation of the more linear polyamide chain structure towards a loosely crosslinked rubbery like structure. In this direction the alkoxysilane end group of the ICTS acts as additional crosslinking point in the system. 

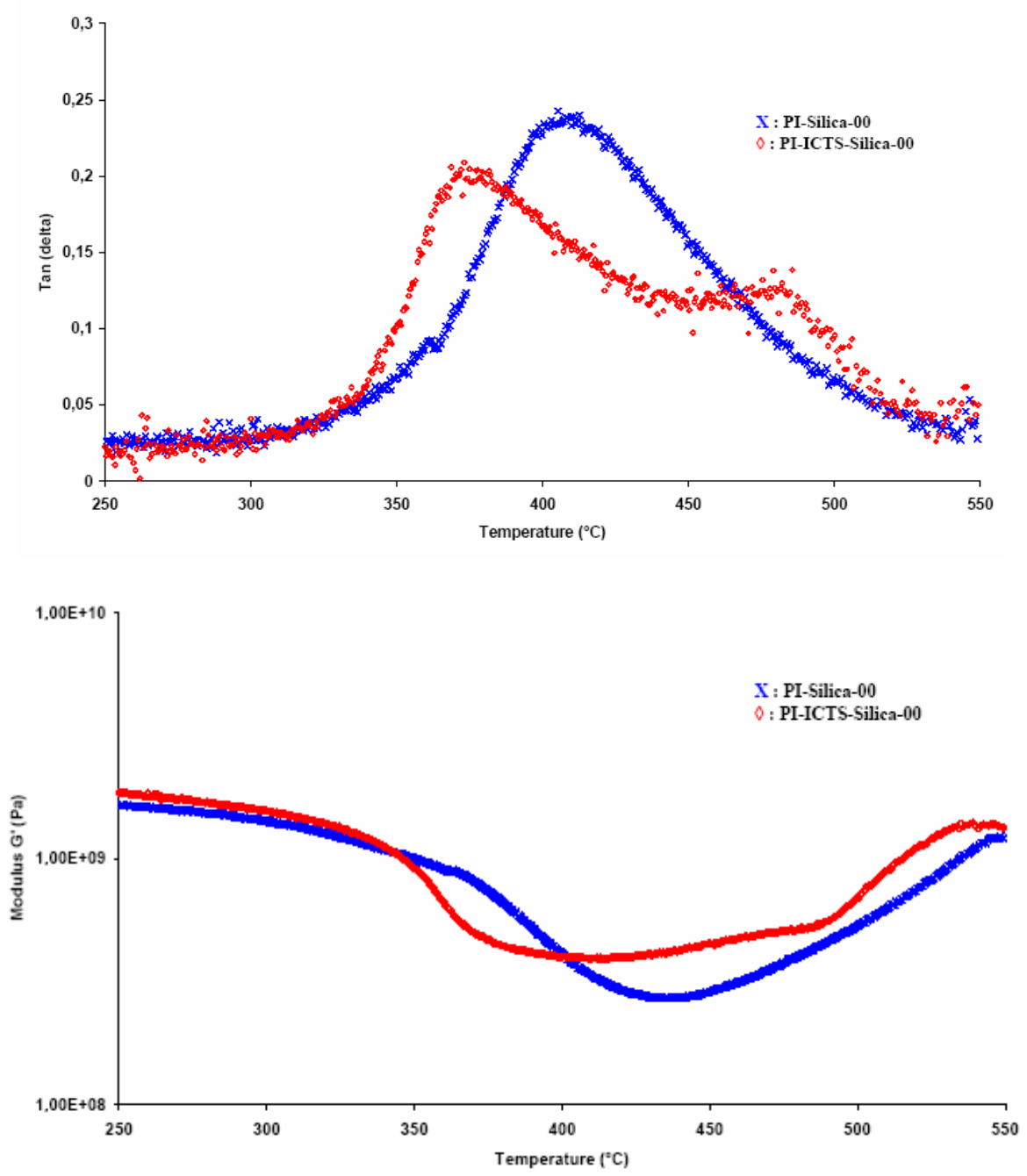

Fig. 6. Dynamic mechanical properties of hybrid films $(x)$ : PI-Silica-00, $(\diamond)$ : PI-ICTSSilica-00.

Tab. 2. Dynamical mechanical properties at $10 \mathrm{~Hz}$ of the PI-Silica and PI-ICTS-Silica hybrid films with different contents of silica.

\begin{tabular}{|c|c|c|c|c|c|}
\hline $\begin{array}{c}\text { Sample } \\
\text { Identification }\end{array}$ & $\begin{array}{l}T_{a 1}\left({ }^{\circ} \mathrm{C}\right) \\
\text { (a) }\end{array}$ & $\begin{array}{l}\mathrm{T}_{\mathrm{\alpha} 2}\left({ }^{\circ} \mathrm{C}\right) \\
\text { (a) }\end{array}$ & $\begin{array}{c}\text { Amplitude of } \\
\tan \delta\left(T_{\alpha 1}\right)\end{array}$ & $\begin{array}{l}\text { Amplitude } \\
\text { of } \tan \delta\left(T_{\alpha 2}\right)\end{array}$ & $\begin{array}{c}E^{\prime}(\mathrm{Mpa}) \times 10^{-6} \\
\text { (b) }\end{array}$ \\
\hline PI-Silica 00 & 408,5 & - & 0.24 & - & 270 \\
\hline PI-Silica 05 & 404 & - & 0.23 & - & 270 \\
\hline PI-Silica 10 & 394 & - & 0.22 & - & 500 \\
\hline PI-Silica 15 & 385 & - & 0.20 & - & 600 \\
\hline PI-ICTS-Silica 00 & 375.5 & 480 & 0.2 & 0.125 & 392 \\
\hline PI-ICTS-Silica 05 & 402 & 503 & 0.17 & 0.115 & 404 \\
\hline PI-ICTS-Silica 10 & 407 & 522 & 0.15 & 0.075 & 620 \\
\hline
\end{tabular}

(a) measured at the maximum of tan $\delta$.

(b) Measured at $\mathrm{Tg}+50^{\circ} \mathrm{C}$. 
The interpretation of the microstructure of the PI-ICTS-Silica00 hybrid film is confirmed by analysing the viscoelasctic properties of PI-ICTS-Silica hybrid films with various amounts of silica filler, presented in Figure 7 . In fact the a2 relaxation is very sensitive to the content of silica in the hybrid film. It shifts toward higher temperature when the content of silica is increased. This observation confirms that such relaxation may be attributed to silica / polymer mixed phase. Concerning the a1 relaxation, it also shifts toward higher temperature when the content of silica in the hybrid film is increased. This trend could be explained by taking into account the microstructure of these hybrid films described above by SAXS and TEM investigations. In fact, the strong interactions between organic and inorganic phases induced by the coupling agent results in a reduction of polyimide chain mobility or flexibility, so that more heating energy is needed to induce the relaxation of polyimide chains.
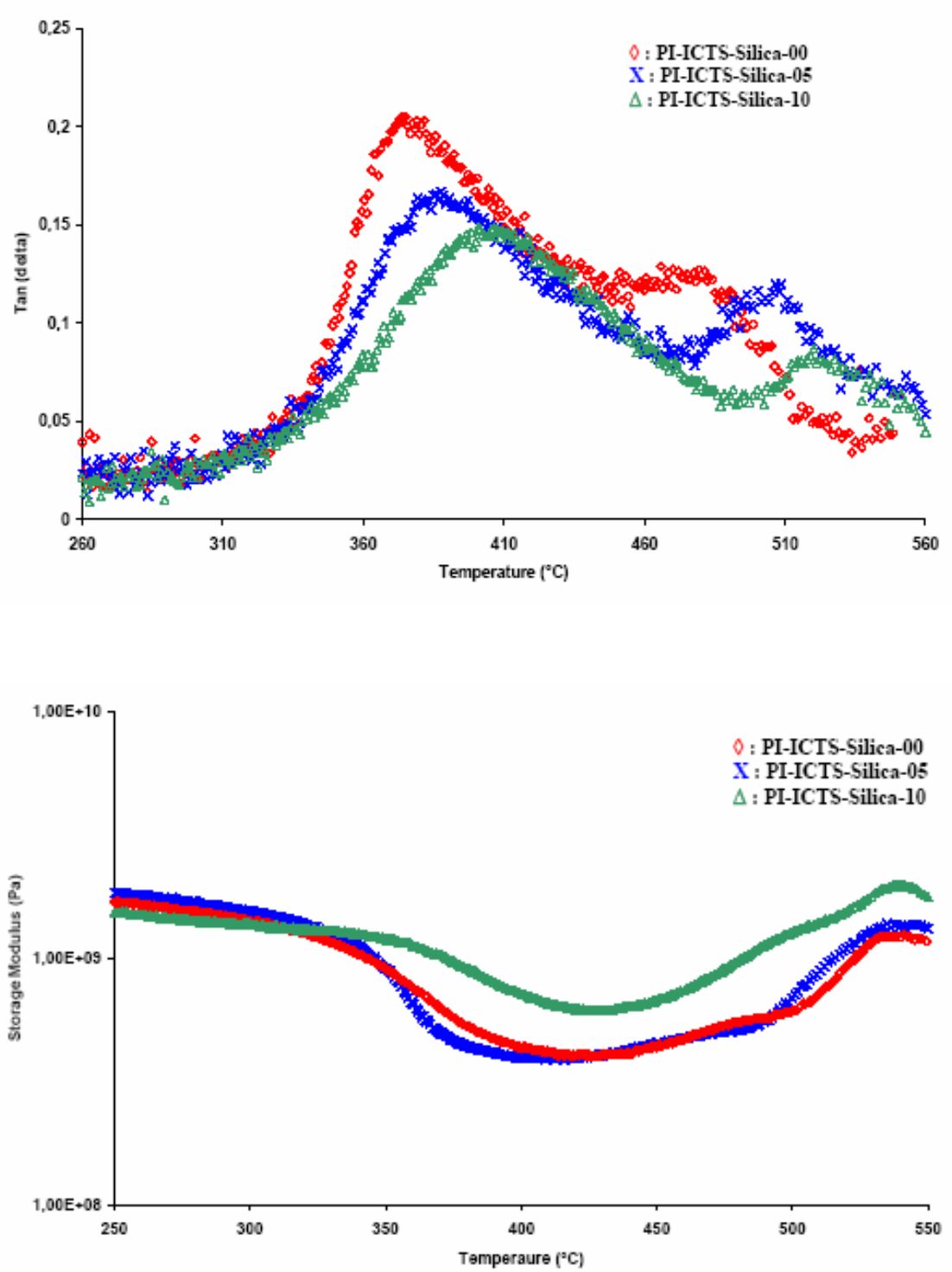

Fig. 7. Dynamic mechanical properties of PI-ICTS-Silica hybrid films with various content of silica $(\diamond)$ : PI-ICTS-Silica 00, (x): PI-ICTS-Silica-05, ( $\Delta)$ PI-ICTS-Silica 10. 
Viscoelastic properties of PI-Silica hybrid films are presented in Figure 8 . Only one relaxation, $\alpha 1$, is observed which is associated with polyimide chains. The micrometric scale phase separation observed by SAXS and TEM is due to the lack of strong interactions (covalent bonds) between the organic and inorganic phases and consequently the absence of mixed phase in such hybrid films. On the other hand the relaxation peak shifts towards lower temperature when the content of silica in the hybrid film is increased which is explained by plastification phenomenon induced by the inorganic particles. This behaviour may be explained by a decrease in network density in the matrix caused by the presence of silica particles having only weak interaction with the polyimide matrix.
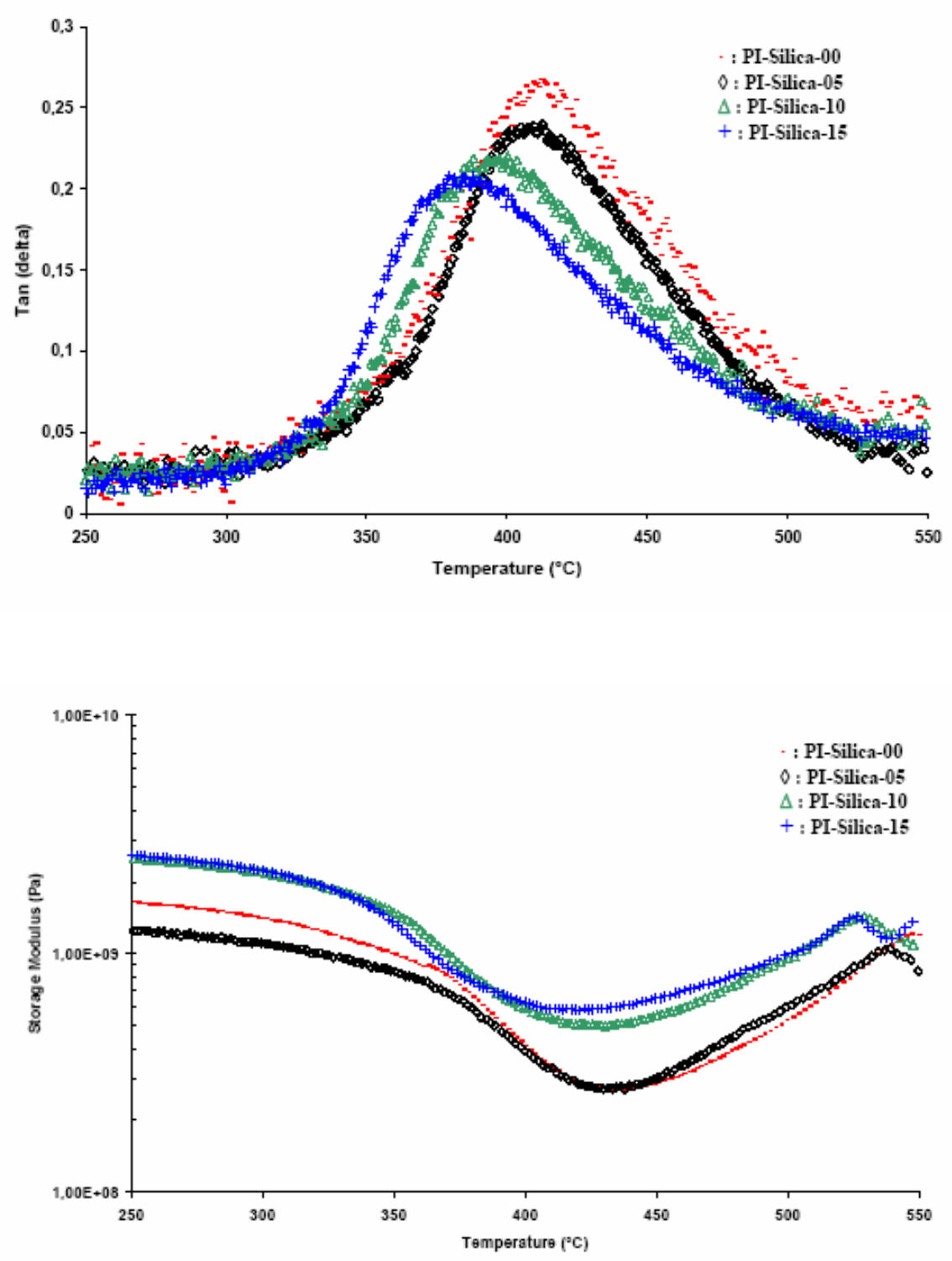

Fig. 8. Dynamic mechanical properties of PI- Silica hybrid films with various content of silica (.): PI- Silica 00, (仓): PI- Silica-05, ( $\Delta$ ) PI- Silica-10, (+) PI- Silica-15. 
For both families of hybrid films the storage modulus at the rubbery like state increases with the content of silica in the hybrid film which is explained by the filler effect of silica. This trend is more pronounced for the PI-ICTS-Silica hybrid films i.e. with coupling agent ICTS. The behaviour could be attributed to two possible chemical phenomena: i) the crosslinking reactions of the silica phase which continue after heating. This phenomenon has been reported in other hybrid materials prepared via the in-situ polymerization of metal alkoxides. ii) Carbonization and / or graphitization of polyimide polymer chains which take place at higher temperature (more than $400^{\circ} \mathrm{C}$ ). This phenomenon is typical for polyimide polymers and is well known in the field of preparation of carbon fibres from polyimide and polyamide polymers [14-16].
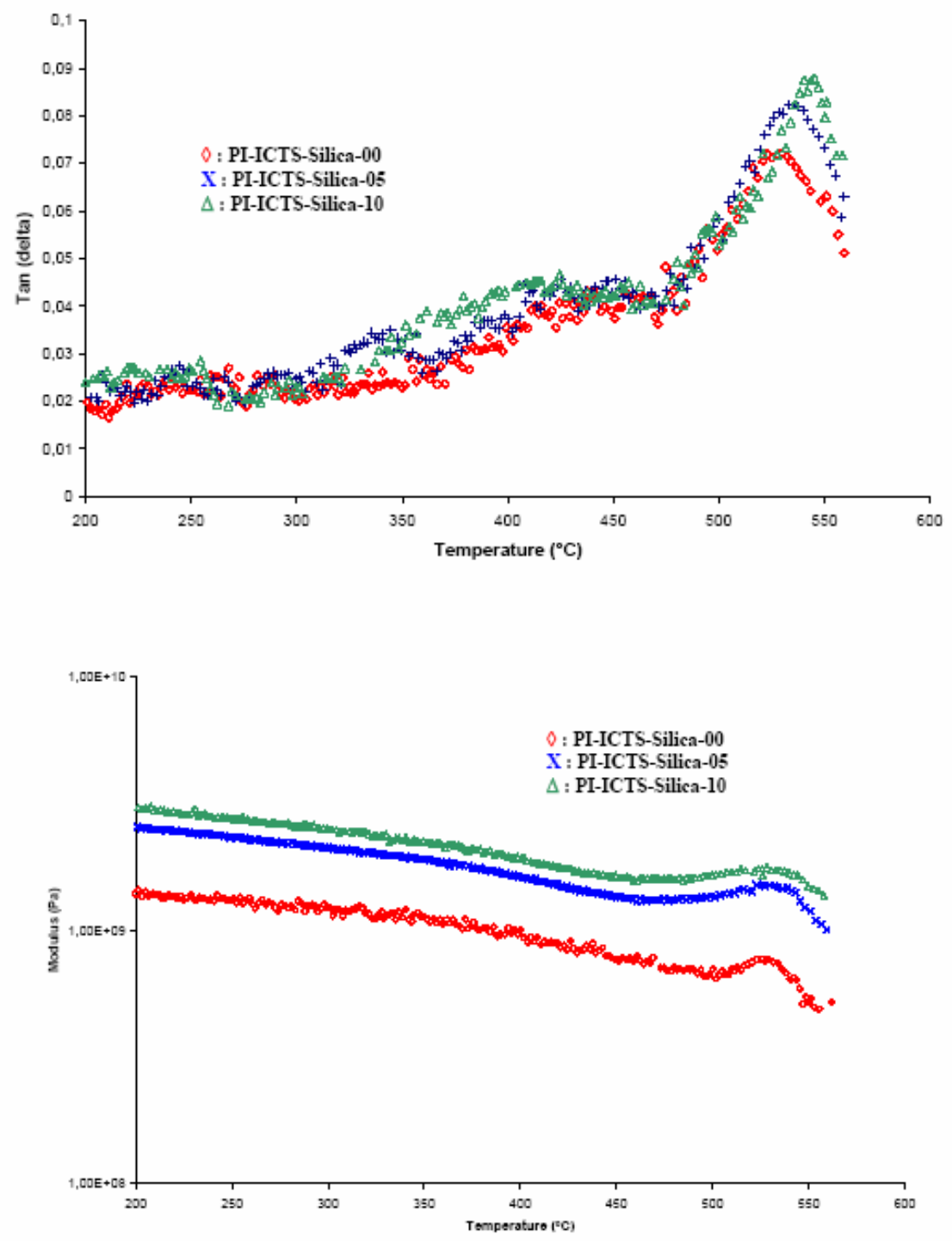

Fig. 9. Dynamic mechanical properties of PI-ICTS-Silica hybrid films with various content of silica post-cured at $450^{\circ} \mathrm{C}$ during 4 hours (x ): PI-ICTS-Silica 00, ( $)$ ): PI-ICTS-Silica-05, ( $\nabla)$ PI-ICTS-Silica 10.

Figure 9 shows the viscoelastic properties of PI-ICTS-Silica hybrid films which undergo post curing at $450^{\circ} \mathrm{C}$ during 4 hours. The most interesting phenomenon to be discussed is the a 1 relaxation around $400^{\circ} \mathrm{C}$ which practically disappeared after 
the post curing process. This phenomenon confirms the latest discussion on the increase of the rubbery like modulus at higher temperature observed in the viscoelastic spectra of the studied hybrid films. So the disappearance of a1 relaxation after post-curing could be explained by carbonization and/or graphitization phenomena on the polyimide chains which results on their transformation to hard graphitic like structure. This is confirmed by the higher modulus values obtained at the rubbery like state. On the other hand the $\alpha 2$ relaxation didn't disappear after post curing but shifted to higher temperature and its amplitude decreased sharply indicating a pronounced cross linking in the systems.

\section{Conclusion}

Combined TEM, SAXS and DMTA investigations on Polyimide / TEOS based hybrid organic - inorganic materials have shown to be an important tool in gaining information about the effects of coupling agent ICTS on the morphology and dynamic mechanical properties of these materials. TEM analysis gives visible information on the size of the inorganic domains in the hybrid materials. SAXS investigations provide information at the nanometer scale. It was shown that silica domain of nanometer size are obtained when coupling agent ICTS is used in the formulation. TEM and SAXS analysis indicate that miscibility of organic and inorganic phase in the hybrid films at the molecular scale is obtained by the addition of ICTS to the polyamic acid. So the addition of ICTS brings a morphological transformation from a dispersed particle microstructure to fine interpenetrated or co-continuous phases. DMTA analysis shows that the strong interaction and miscibility between organic and inorganic components have important influences on the dynamic mechanical properties of the resulting hybrid systems indicated by different relaxation behaviour. Higher values of the rubbery modulus and lower relaxation temperature are obtained for the hybrid materials with coupling agent ICTS. On the other hand a mixed phase was evidenced by DMTA analysis which has higher relaxation temperature. DMTA analysis also shows graphitization and / or carbonization phenomenon of the polyimide chains that occur at higher temperature which considerably change the mechanical and thermal properties of the hybrid materials.

\section{Experimental part}

Materials

4,4'-Oxydianiline (ODA) and Pyromellitic dianhydride (PMDA) were purchased from Riedel-deHaen, 2,2-Bis(3-amino-4-hydroxyphenyl) hexafluoro-propane (6F-OHDA) is supplied by Fluorochem. Tetraethoxysilane (TEOS) and N,N-Dimethylacetamide (DMAc) are supplied by Aldrich, 3-Isocyanatopropyltriethoxysilane (ICTS) is supplied by $A B C R$, Hydrochloric acid from Fluka. All reagents and solvents were used without further purification.

\section{Preparation of polyamic acids}

Two types of polyamic acids were synthesised: unmodified and modified with the coupling agent ICTS. For both polyamic acids the preparation has been made by the co-polymerization of a mixture of diamines, ODA and appropriate amount of 2,2Bis(3-amino-4-hydroxyphenyl)hexafluoro-propane (6F-OHDA) with PDMA in DMAc. 
The molar ratio ODA/ 6F-OHDA has chosen to be $90: 10$ and the molar ratio of diamine $/$ dianhydride $=1$.

For both polyamic acids the mixture of diamines in DMAc was stirred at $20^{\circ} \mathrm{C}$ for 15 min under nitrogen atmosphere in order to ensure complete mixing. Subsequently the PMDA was added at once to the stirring mixture. After 1 hour of stirring, viscosity of the polyamic acid was found to increase sharply. To facilitate stirring the reaction mixture was again diluted with DMAc and stirred for further $18 \mathrm{~h}$ for completing the reaction.

The modification of polyamic acid with ICTS was done by drop wise addition of a solution of appropriate amount of silane in DMAc (verifying the molar ratio ICTS/6F$\mathrm{OHDA}=1.5$ ). Solution was allowed to react at $20^{\circ} \mathrm{C}$ for $6 \mathrm{~h}$ and subsequently at $60^{\circ} \mathrm{C}$ for $3 \mathrm{~h}$.

From these two polyamic acids two families of hybrid organic-inorganic materials were prepared by in-situ hydrolysis and condensation of TEOS. The first family was denoted PI-silica and the second one PI-ICTS-silica. For both families, the required amount of TEOS was mixed with the polyamic acid solution in order to obtain 5, 10 15 and $30 \%(\mathrm{w} / \mathrm{w})$ of silica in the matrix respectively. In-situ hydrolysis and condensation was carried out by adding the stoichiometric amount of water (as $20 \%$ $\mathrm{w} / \mathrm{w}$ of $0.1 \mathrm{M} \mathrm{HCl}$ in DMAc) required.

The resulting mixture was stirred at $60^{\circ} \mathrm{C}$ for $12 \mathrm{~h}$. Thin films were then cast on Teflon Petri dishes. Solvent solution were carried out at $70^{\circ} \mathrm{C}$ for $12 \mathrm{~h}$ and then at $80^{\circ} \mathrm{C}$ for additional $4 \mathrm{~h}$. Imidization was carried out by successive heating at $100^{\circ} \mathrm{C}$, $200^{\circ} \mathrm{C}$ and $300^{\circ} \mathrm{C}$ for each $1 \mathrm{~h}$ under nitrogen. Figure 1 illustrates a schematic diagram of the preparation methods of PI-silica and PI-ICTS-silica hybrid films.

\section{Characterisation}

For the investigation of the morphology of the samples by transmission electron microscopy (TEM), the nanocomposites were ultramicrotomed using a Reichert ultra cut with a diamond knife. The resulting slices, with an approximate thickness of 100 $\mathrm{nm}$, were prepared on 400 mesh copper grid. The dispersion of silica particles was directly visible in TEM without further preparation.

Small angle X-ray scattering (SAXS) measurements were performed using compact kratky camera (Paar (Graz), type KKK) with slit collimation and equipped with a linear position sensitive detector (Braun, 2048 channels). The $\mathrm{X}$-ray wave length was 0.154 nm using monochromatized vectors $q$ with $q=\frac{4 \pi}{\lambda \cdot \sin (\theta)}$ were $2 \theta$ is the scattering angle, range from $q=0.035 \mathrm{~nm}^{-1}$ to $5 \mathrm{~nm}^{-1}$. The SAXS spectra have been corrected for parasitic background scattering and for the background contribution of the organic polymer phase.

The dynamic mechanical thermal properties were measured using computer controlled Rheometric $V$ Dynamic Viscoelastometer in the tensile mode. A frequency of $10 \mathrm{~Hz}$ was used to study the temperature dependence of the dynamic mechanical properties (storage modulus $E^{\prime}$, loss modulus, E', and loss factor, $\tan \delta$ ). All measurements were carried out at a heating rate of $2^{\circ} \mathrm{C} / \mathrm{min}$. Typical dimensions of 
the sample used were $15 \mathrm{~mm}$ in length, $6 \mathrm{~mm}$ in width and $0.15-0.20 \mathrm{~mm}$ in thickness.

\section{References}

[1] Feger, G.; Khojasteh, M. M.; McGrath; J. E.; Polyimides: Materials, Chemistry and Characterization, Elsvier, Amsterdam, 1989.

[2] Numata, S. and Kinjo, N.; Polym. Eng. Sci. 1988., 28, 906.

[3] Sanchez, C.; and Ribot, F.; New J. Chem. 1994, 1007.

[4] Wang, B. and Wilkes, G.; Pure Appl. Chem. 1994, A 31, 249.

[5] Kasemann, R.; and Schmidt, H.; in "First European Workshop on Hybrid Organic Inorganic Materials: Synthesis, Properties, Applications" (Château de Bierville, France) 1993, 171.

[6] Kaddami, H.; Surivet, F.; Gérard, J. F.; Lam, T. M.; and Pascault, J. P.; J. Inorg. Organomet. Poly. 1994, 4, 183.

[7] Landry, G. J. T.; Coltrain, B. K.; and Brady, B. K.; Polymer 1992, 33, 1486.

[8] Becker, C.; Krug, H. and Schmidt, H.; Mat, Res. Soc. Symp. Proc. 1996, 237, 435.

[9] Coltrain, B. K.; Landry, C. J. T.; O’Reilly, J. M.; Chamberlain, A. M.; Rakes, G. A.; Sedita, J. S.; Kelts L.W.; Landry, M. R. and Long, V. K.; Chem. mat. 1993, 5(10), 1445.

[10] Landry, C. J. T.; Coltrain, B. K.; Landry, M. R.; Fitzgerald, J. J. and Long, V. K.; Macromolecules 1993, 26, 3702.

[11] Hajji, P.; Kaddami, H.; Gérard, J. F.; David, L.; Pascault, J. P. and Vigier, G.; in "Better Ceramics Through Chemistry", ed.: Coltrain, B. K.; Sanchez, C. and Wilkes, G.; MRS Publ. 1999, 576, 357.

[12] Kaddami, H.; Gérard, J. F.and Pascault, J. P.; in "Better Ceramics though Chemistry X", Ed.: Coltrain, B. K.; Sanchez, C. and Wilkes, G.; MRS Publ. 1999, $576,51$.

[13] Huang, H. H. and Wilkes, G.L.; Polymer Bulletin 1987, 18, 455.

[14] Inagaki, M.and Hishiyama, K.,I. S. Y.; J. Mater. Res. 1996, 6(5), 1108.

[15] Fujio, O; Shinji, K; Hidekazu, T.; Hai, L.; Mutsumi, K. and Hirofusa, Sh.; Syn. Met. 2001, 125(2), 197.

[16] Takeichi, T.; Eguchi, Y.; Kaburagi, Y.; Hishiyama, Y. and Inagaki, M.;Carbon 1999, 37 (4) 569. 